\title{
Relation entre les symptômes de fusariose de l'épi et la perte de rendement chez le blé tendre
}

\author{
L Saur 1, N Benacef 2 \\ avec la collaboration technique de JY Morlais \\ IINRA, SRIV, BP 29; \\ 2 INRA, station d'amélioration des plantes, BP 29, F-35650 Le Rheu, France
}

(Reçu le 11 mars 1993; accepté le 14 septembre 1993)

\begin{abstract}
Résumé - Durant 4 saisons culturales, des essais ont été réalisés au champ sur 4 variétés de blé d'hiver en contamination artificielle sur épi par Fusarium roseum var culmorum, avec différentes concentrations de spores à différents stades physiologiques de l'hôte. Le développement de la maladie a été évalué en végétation par le pourcentage d'épillets desséchés au début du stade grain pâteux, et à la récolte par le pourcentage de grains de couleur blancrose (grains fusariés). L'effet de la maladie sur le rendement a été mesuré par référence à un témoin non contaminé. II existe une relation linéaire entre les symptômes sur grain et les notations en végétation, mais un effet de l'année et de la variété est mis en évidence. Le pourcentage de grains fusariés donne une meilleure estimation de la perte de rendement que les symptômes en végétation, avec un effet de l'année moins important. Deux variétés montrent une courbe de réponse non linéaire dans la relation entre la diminution de poids de 1000 grains et les symptômes sur grains. L'utilisation des notations en végétation pour évaluer la gravité de la maladie et la notion de tolérance des variétés sont discutées.
\end{abstract}

\section{Fusarium roseum var culmorum / blé / rendement / cultivar}

Summary - Relationship between head blight symptoms of Fusarium roseum var culmorum and yield losses in wheat. Four winter wheat cultivars were inoculated in field tests by Fusarium roseum var culmorum over a 4-yr period. Several spore concentrations of the inoculum were used each year. The severity of the disease was evaluated using the number of damaged spikelets at the early dough growth stage and the percentage of visually infected seeds at harvest. Yield and 1000 grain weight losses were measured with reference to an uninoculated control. A significant correlation was found between the number of damaged spikelets and the number of visually infected seeds together with an effect of the variety and the year. On a linear basis, because of a weak year effect, the decrease in 1000 grain weight is better predicted using the number of visually infected seeds than the number of damaged spikelets. There is a trend for non-linear response curves for 2 varieties. The use of field evaluation of severity of the disease and tolerance of varieties are discussed.

Fusarium roseum var culmorum / wheat / yield / cultivar

\section{INTRODUCTION}

La fusariose de l'épi provoque des baisses de rendement très importantes chez le blé dans les régions à été chaud et humide : Est du Canada, région du Yangtzé en Chine (Cook, 1981 ; Martin et Johnson, 1982). En France, de fortes attaques sont signalées lorsqu'une période pluvieuse intervient à la floraison et durant la période de rem- plissage du grain. Cette maladie se manifeste par le dessèchement précoce de certains épillets. Les grains issus des épillets contaminés sont envahis par le parasite et prennent une coloration blanche ou rose. La gravité de l'attaque peut être appréciée par le pourcentage d'épillets présentant des symptômes. Cette notation, délicate, doit être réalisée lorsque la maladie a atteint son plein développement mais avant que le 
dessèchement dû à la maturité des pièces florales ne soit commencé. Les observations portant sur les grains donnent une idée du développement de la maladie à la fin du cycle de la plante. Elles peuvent consister en la détection de la présence du parasite par isolement sur milieu nutritif (Schroeder et Christensen, 1963; Mesterhazy, 1978) ou le dénombrement des semences ne donnant pas de plantule dans un essai de germination (Leijerstam, 1962 ; Mesterhazy, 1978). Chupin et al (1988) proposent d'évaluer l'importance de la maladie par le pourcentage de grains présentant l'aspect caractéristique de l'envahissement par le parasite. II existe cependant peu de références concernant l'appréciation des dégâts causés par la fusariose de l'épi à partir des symptômes. Le but de cette étude est de comparer deux méthodes d'évaluation de la gravité de la maladie en relation avec la perte de rendement, en essayant de dégager l'influence de l'année et de la variété.

\section{MATÉRIEL ET MÉTHODES}

\section{Dispositifs}

De 1985 à 1988, des essais au champ ont été mis en place avec contamination artificielle sur 4 variétés de blé d'hiver: Talent, Fidel, Arminda et Rescler. Afin d'obtenir différents niveaux de maladie, plusieurs concentrations en spores de l'inoculum ont été utilisées dans chaque essai. Des témoins non contaminés ont été ajoutés dans le but de mesurer l'effet de la maladie sur le rendement. Les nombres de traitements effectués chaque année sont donnés dans le tableau I. En 1985 et 1986, des essais supplémentaires ont été réalisés afin d'étudier plus particulièrement les réactions de la variété sensible Rescler.

L'inoculum était constitué d'une suspension de spores dans de l'eau obtenue à partir d'une culture de Fusarium roseum var culmorum (Schwabe) $\mathrm{Sn}$ et $\mathrm{H}$ sur grain d'orge humide stérilisé. Les conidies ont été récupérées par lavage à l'eau, et la concentration de la suspension ajustée après titration à l'hématimètre. Pour chaque essai, l'inoculum correspondant aux différents traitements a été obtenu par dilution successive au demi ou au tiers d'une suspension de base de $10^{6} \mathrm{spores} / \mathrm{ml}$ ou $2 \times 10^{6}$ spores $/ \mathrm{ml}$.

La contamination a été effectuée par pulvérisation à l'anthèse de chaque variété sauf pour 3 traitements de l'essai $n^{\circ} 2$ de 1986 , qui ont été contaminés respectivement aux stades épiaison, floraison plus $3 \mathrm{j}$ et floraison plus $10 \mathrm{j}$. Afin d'assurer des conditions favorables à l'installation du parasite, les contaminations ont été réalisées le soir après une irrigation par aspersion.
Tableau I. Nombre de traitements réalisés dans les essais au champ de 1985 à 1988 , en contamination artificielle par Fusarium roseum var culmorum, avec différentes concentrations en spores de l'inoculum à différents stades physiologiques du blé.

\begin{tabular}{cllllll} 
Variété & \multicolumn{2}{c}{1985} & \multicolumn{2}{c}{1986} & 1987 & 1988 \\
& Essai & Essai & Essai & Essai & & \\
& $n^{\circ} 1$ & $n^{\circ} 2$ & $n^{\circ} 1$ & $n^{\circ} 2$ &
\end{tabular}

$\begin{array}{lllllll}\text { Rescler } & 3^{1} & 8 & 3 & 16 & 8 & 9 \\ \text { Talent } & 3 & - & 3 & - & 8 & 9 \\ \text { Fidel } & 3 & - & 3 & - & 8 & 9 \\ \text { Arminda } & 3 & - & 3 & - & 8 & 9\end{array}$

1 Nombre de traitements, y compris le témoin non contaminé.

Le dispositif expérimental était de type bloc pour les essais à une variété ou de type split-plot pour les essais à plusieurs variétés, avec 4 répétitions. La parcelle élémentaire était formée de 4 lignes de $1,5 \mathrm{~m}$ espacées de $20 \mathrm{~cm}$.

\section{Notations et mesures}

La notation des symptômes en végétation a été effectuée 350 degrés j environ après la contamination, soit 18 à 28 j selon l'année et la variété. Cette période correspond au milieu ou à la fin du palier hydrique du grain et au début du stade grain pâteux. Le pourcentage d'épillets présentant des symptômes (épillets fusariés : EF) a été mesuré par dénombrement sur 50 épis par parcelle.

Un échantillon de 50 épis prélevé au hasard a été récolté dans chaque parcelle. Le battage a été réalisé à l'aide d'une batteuse de laboratoire avec une ventilation faible. Le poids de grain total et le poids de 500 grains ont été mesurés. Les grains présentant la couleur blanc rose caractéristique de l'envahissement par F roseum (grains fusariés : GF) ont été dénombrés sur un échantillon de 500 grains.

\section{RÉSULTATS}

Le pourcentage d'épillets fusariés était nul ou très faible dans les témoins non contaminés avec un maximum de $0,3 \%$ pour les variétés Rescler et Talent en 1987. La contamination artificielle avec l'inoculum de concentration la plus élevée s'est traduite chaque année par un bon développement de la fusariose de l'épi. Pour les autres traitements, l'intensité de la maladie était 
d'autant plus faible que l'inoculum était plus dilué. L'attaque était plus faible pour les contaminations avant ou après la floraison que pour les contaminations à l'anthèse. Si l'on considère les résultats des années 1987 et 1988 pour lesquelles les 4 variétés ont été soumises aux mêmes modalités de contamination, la variété Rescler se distingue des 3 autres par sa plus grande sensibilité au parasite. Sur l'ensemble des 4 années d'expérimentation, nous disposons d'une gamme d'attaque par la fusariose de l'épi qui varie de 0 à $47 \%$ d'épillets fusariés pour la variété Rescler, $18 \%$ pour Arminda, $15 \%$ pour Fidel et $11 \%$ pour Talent (fig 1).

\section{Relation entre les symptômes sur grain et les symptômes en végétation}

Le coefficient de corrélation entre le pourcentage d'épillets desséchés et le pourcentage de grains fusariés à la récolte est significatif et sa valeur est élevée $(r=0,811$ pour $113 \mathrm{dl})$. Les pentes des droites de régression sont différentes selon les variétés avec une pente plus élevée pour la variété Talent (tableau II).

L'observation de la figure 1 montre que, pour Rescler, l'année 1986 se distingue des autres années par un nombre de grains fusariés inférieur, à symptômes sur épillets égaux. Cette année-là, la notation pour Rescler a eu lieu après $3 \mathrm{j}$ de température maximum proche de $30^{\circ} \mathrm{C}$. II est probable que, dans cette situation, des dessèchements de pièces florales qui ne se produisent pas en année normale à ce stade se soient manifestés. Pour Arminda et, dans une moindre mesure, Fidel, ce sont les résultats de l'année 1988 qui s'écartent de la relation. Pour Talent, les différentes années ne peuvent être distinguées.

Tableau II. Coefficient de corrélation $r$ et pente de la droite de régression $b$ entre le pourcentage de grains fusariés à la récolte (GF) et le pourcentage d'épillets atteints au stade grain pâteux (EF).

Rescler Talent Fidel Arminda

$\begin{array}{lcccc}r & 0,825 & 0,876 & 0,891 & 0,819 \\ b & 1,11 & 3,86 & 2,10 & 2,29 \\ & \pm 0,22 & \pm 0,89 & \pm 0,48 & \pm 0,72 \\ \text { Degrés de liberté } & 44 & 21 & 21 & 21\end{array}$

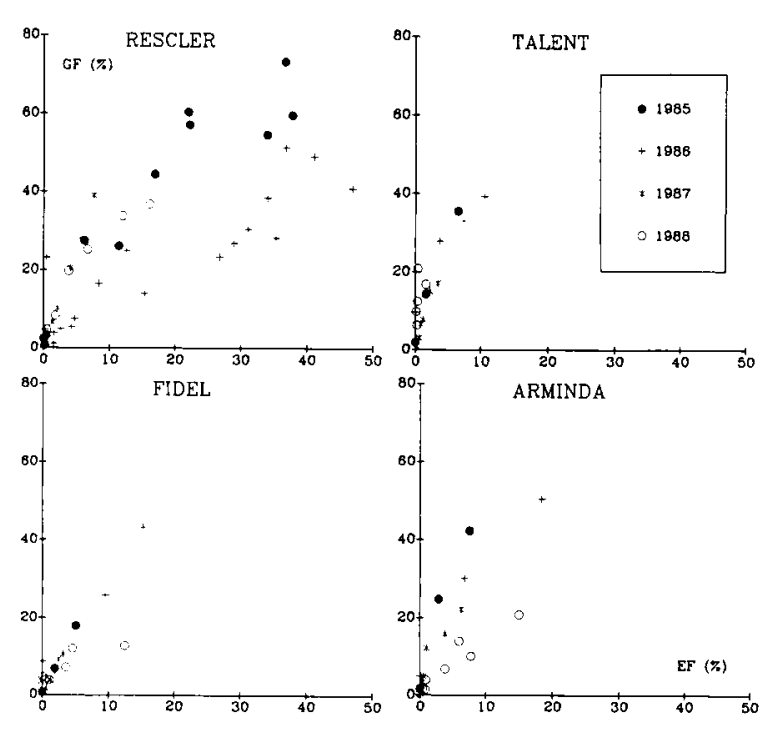

Fig 1. Relation entre le pourcentage de grains fusariés à la récolte (GF) et le pourcentage d'épillets présentant des symptômes au stade grain pâteux (EF).

Un des traitements de l'essai $n^{\circ} 2$ de 1986 (variété Rescler) présente $23 \%$ de grains fusariés pour très peu de symptômes. II s'agit de la contamination $10 \mathrm{j}$ après la floraison pour laquelle les symptômes n'étaient pas extériorisés au moment de la notation.

\section{Relation entre les symptômes et la perte de rendement}

Étant donné la grande hétérogénéité entre répétitions due à l'irrégularité du peuplement pour les mesures portant sur le rendement en 1987, les résultats concernant cette année n'ont pas été inclus dans l'interprétation.

Les 2 composantes du rendement affectées par la fusariose de l'épi sont le poids de 1000 grains et le nombre de grains par épi. Sur l'ensemble de nos résultats, la diminution moyenne du poids de 1000 grains des parcelles contaminées par rapport aux parcelles témoins est de $13,6 \%$ alors que la diminution du poids de grain par épi est de $16,9 \%$. Une proportion de $20 \%$ environ de la diminution de rendement peut donc être attribuée à la diminution du nombre de grains par épi.

Le coefficient de corrélation entre la diminution du poids de 1000 grains et la diminution du poids de grain par épi est élevé $(r=0,958$ pour $81 \mathrm{dl})$. Or, sur de petites parcelles comme celles 
utilisées dans nos essais, la précision de la mesure du poids de grain par épi est inférieure à celle du poids de 1000 grains. Dans ces conditions, nous utiliserons la diminution du poids de 1000 grains par rapport au témoin non contaminé exprimée en pourcentage (DPMG\%), pour étudier l'effet de la fusariose sur le rendement.

Le coefficient de corrélation entre les symptômes et la diminution relative de poids de 1000 grains est plus élevé pour les notations sur grains ( $r=0,911$ pour $81 \mathrm{dl}$ ) que pour les notations en végétation ( $r=0,838$ pour $81 \mathrm{dl}$ ). Toutes variétés et toutes années confondues, on peut estimer la perte de poids de 1000 grains due à la fusariose à partir du pourcentage de grains fusariés :

$$
\text { DPMG } \%=0,83 \mathrm{GF}+1,49 .
$$

Puisque la perte de poids de 1000 grains causée par la fusariose représente environ $80 \%$ de la perte totale de rendement, la relation entre le pourcentage d'épillets fusariés et la perte de rendement exprimée en pourcentage a une pente voisine de 1.

II existe des différences entre les pentes des droites de régression entre les symptômes et la diminution du poids de 1000 grains en fonction de la variété, surtout en ce qui concerne les symptômes en végétation (tableau III). Comme dans le cas de la relation entre les symptômes sur grains et les symptômes en végétation, Talent se distingue de Rescler, Fidel et Arminda pour la relation entre la diminution du poids de

Tableau III. Coefficient de corrélation $r$ et pente de la droite de régression $b$ entre la diminution relative de poids de 1000 grains (DPMG\%) et le pourcentage d'épillets atteints au stade grain pateux (EF) ou le nombre de grains fusariés à la récolte (GF).

Rescler Talent Fidel Arminda

$\begin{array}{lccccc}\text { DPMG\%/EF } & r & 0,815 & 0,927 & 0,760 & 0,779 \\ & b & -0,96 & -3,53 & -1,08 & -1,28 \\ & \pm 0,22 & \pm 0,86 & \pm 0,56 & \pm 0,62 \\ & & & & & \\ & b & -0,83 & -0,82 & -0,50 & -0,53 \\ & \pm 0,08 & \pm 0,26 & \pm 0,22 & \pm 0,15 \\ & & & & \\ \begin{array}{l}\text { Degugés } \\ \text { de liberté }\end{array} & 36 & 13 & 13 & 13\end{array}$

1000 grains et le pourcentage d'épillets fusariés : pour cette variété, les symptômes en végétation ne reflètent pas dans les mêmes proportions que pour les autres variétés les dégâts sur les grains et la perte de rendement.

L'observation de la distribution des points dans la relation entre le pourcentage de grains fusariés et la diminution relative du poids de 1000 grains (fig 2) montre que l'effet année est faible. Les points sont remarquablement alignés pour les variétés Rescler et Talent. La relation est moins linéaire pour Fidel et Arminda : pour les niveaux d'attaque faibles, la relation se confond avec celle de Rescler et Talent; pour les niveaux plus forts, au-dessus de 30\% de grains fusariés, la courbe s'infléchit. Dans ce dernier cas, la perte de rendement est inférieure à celle observée pour les 2 autres variétés.

\section{DISCUSSION}

L'observation des grains à la récolte permet une bonne évaluation de l'effet de la fusariose sur le rendement. Pour les 4 variétés étudiées, sur les 4 campagnes culturales, la perte de rendement due à la fusariose de l'épi peut être estimée à partir du dénombrement des grains couverts de mycélium à la récolte. Cette constatation rejoint les conclusions de Chupin et al (1988) obtenues à partir de résultats de plusieurs lieux sur deux années dans des essais fongicides. La pente de la droite de régression de la diminution relative du poids de grain par épi sur le pourcentage de

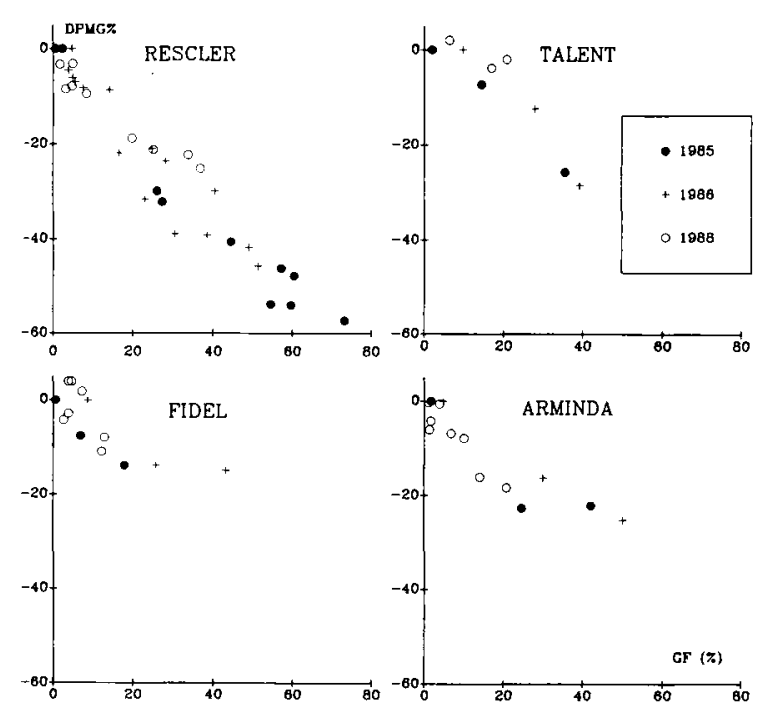

Fig 2. Relation entre la diminution relative du poids de 1000 grains (DPMG\%) et le pourcentage de grains fusariés à la récolte (GF). 
grains fusariés est voisine de un pour les 2 études.

Deux facteurs peuvent expliquer la bonne correspondance entre la quantification visuelle de symptômes et la perte de rendement dans le cas de la fusariose de l'épi :

- la durée et la position de la maladie dans le cycle de la plante : pour ce qui concerne l'attaque de l'épi qui nous intéresse dans cette étude, le parasite n'est présent sur la plante que pendant une période très courte, de la floraison à la maturité, et dans une phase critique du cycle de la plante pour l'élaboration de 2 importantes composantes du rendement : le nombre de grains et le poids de 1000 grains ;

- la localisation de l'agent pathogène sur la plante : le parasite affecte de très près l'organe récolté, le grain, soit directement en envahissant le caryopse en formation, soit indirectement en bloquant l'alimentation de la partie supérieure de l'épi.

L'évaluation des pertes de rendement à partir des symptômes est moins aisée dans le cas d'une maladie du pied comme le piétin verse, qui se développe dès la levée, ou dans le cas de maladies foliaires, qui agissent indirectement sur le rendement en grain par diminution de la surface photosynthétique ou modification de la physiologie de la plante.

Les notations en végétation posent le problème de la précision des observations. On sait en effet qu'il est facile dans certains cas de surestimer les symptômes, en particulier de considérer comme envahis par le pathogène des épillets dont certaines pièces florales commencent à se dessécher pour des raisons sans rapport avec la présence de l'agent pathogène dans l'épi. Pour établir une bonne relation entre les symptômes et la perte de rendement seuls les épillets réellement envahis par le parasite, dans lesquels on trouve les grains fusariés, doivent être pris en compte.

La plupart de nos contaminations ont été réalisées à la floraison. Pour la contamination tardive, les symptômes n'étaient pas exprimés au moment de la première notation alors que l'on retrouve à la récolte un nombre important de grains fusariés et la perte de rendement correspondante.

Le jugement des génotypes en végétation est indispensable dans un programme de sélection lorsque le choix doit intervenir avant la récolte. Dans ce cas, les contaminations doivent être réalisées au même stade pour tous les génotypes et les notations en végétation doivent être effectuées suffisamment tard mais avant que le des- sèchement physiologique ne masque les symptômes. Les observations des grains après la récolte, plus coûteuses en travail, sont particulièrement intéressantes lorsque l'on souhaite avoir une connaissance précise du matériel végétal.

La réaction de Fidel et Arminda aux contaminations fortes fait penser à un phénomène de tolérance. On sait qu'il peut exister des différences entre variétés pour l'envahissement du rachis à partir d'un épillet attaqué (Schroeder et Christensen, 1963; Saur, 1984) et que le blocage des vaisseaux conducteurs provoque l'échaudage de la partie supérieure de l'épi. Le phénomène de tolérance observé pour Fidel et Arminda pourrait s'expliquer par une résistance à l'envahissement du rachis qui aurait pour conséquence de réduire la diminution du poids de 1000 grains causée par la fusariose de l'épi.

\section{REMERCIEMENTS}

Ce travail a été réalisé dans le cadre de l'action thématique programmée "Écophysiologie du blé winancée par I'ONIC et le ministère de la Recherche et de la Technologie.

\section{RÉFÉRENCES}

Chupin B, Dawson M, Daguenet $G$, Jugnet MP, Genet JL, Lagarde R, Magendie H, Michel F, de Saint Blanquat A, Saur L (1988) Évaluation des dégâts causés par la fusariose (Fusarium roseum LINK) de l'épi chez le blé. CR colloque ANPP. Bordeaux, nov 1988, 211-219

Cook RJ (1981) Fusarium diseases in the people's republic of China. In: Fusarium diseases, biology and taxonomy (PE Nelson, TA Toussoun, RJ Cook, eds) Pennsyl State Univ Press, 53-55

Leijerstam B (1962) Studies on a method of testing, resistance of wheat varieties to fusarial head blight. Statens vâxtskyddsant Medd 12, 90, 182-203

Martin RA, Johnston HW (1982) Effects and control of Fusarium diseases of cereal grains in the Atlantic Provinces. Can J Plant Pathol 20, 210-216

Mesterhazy A (1978) Comparative analysis of artificial inoculation methods with Fusarium spp on winter wheat varieties. Phytopath Z 93, 12-25

Saur L (1984) Comportement de quatre variétés de blé tendre vis-à-vis de la fusariose de l'épi causée par Fusarium roseum var culmorum (Schwabe) Sn et $\mathrm{H}$. agronomie 4, 939-943

Schroeder HW, Christensen JJ (1963) Factors affecting resistance of wheat to scab caused by Gibberella zeae (Schw) Petch. Phytopathology 53, 831838 